\title{
A remarkable simple fluorescent cerium-based chemosensor for selective phosphate detection in aquaeous medium
}

\author{
Thibaud Rossel $^{* \dagger}$ \\ Gymnase français de Bienne, Pré Jean-Meunier 1, Moutier, 2740, Switzerland
}

\begin{abstract}
Phosphates anions are important molecules for the society in general and involved for example in medicine or agriculture. Detection of them with chemosensor is a challenge due to the chemical properties of the anions. In this context, an exquisite chemosensor would have exquisite affinity, selectivity and low detection limit. Here we address this problem using a simple fluorescent indicator displacement assay (FID) with only commercially available chemicals used to probe phosphate ions in aqueous medium using cerium ammonium nitrate (CAN). The monomeric homoleptic complex detects phosphate ions in low millimolar concentrations either spectrophotometrically or with the naked-eye with high selectivity over other anions and high affinity. To our knowledge, this is the first description of a simple sensitive, selective and high affinity cerium-based chemosensor for the fluorescent selective naked-eye detection of phosphate in aqueous medium. It proved useful for the detection of phosphate in Coca Cola $@$.
\end{abstract}

\section{Introduction}

Phosphates are fundamental molecules involved in many key processes such as for example: water treatment, agriculture, medicine. [1-3] Therefore an easy to use, cheap, selective and sensitive analytical method to detect them is highly needed. [3] In this context, the development of remarkable chemosensors requires a low detection limit, high affinity and selectivity for the analytes such as in the case of antibodies for example. [3, 4] For phosphate, it is very difficult to achieve due to the large size of the anion, its hydrophilicity, its multiple protonation states and the Hofmeister series, especially at physiological pH. $[2,5]$

Most of the chemical receptors described in the literature present a combination of these three qualities but rarely the three together. [3, 4, 6-8] The exquisite detection of analytes depends notably on the interactions of the receptor and the analytes but also on

\footnotetext{
*Corresponding author.

${ }^{\dagger}$ E-mail: thibaud.rossel@emsp.gfbienne.ch
} 
the reporter for the detection of it such as in the case of IDAs. [4, 6, 7] The reporter has intrinsic optical properties that dictates the emitted signal analyzed and the signal-to-noise ratio. [7]

Numerous chemosensors for the detection of anions based on displacement assay (DA), have been reported, but most of them use colorimetric indicators for the nakedeye detection of the analytes such as phosphate (IDAs). [2, 6, 7, 9] For example, we reported on such receptors for colorimetric detection of phosphate with a dinuclear cerium inorganic complexes. $[2,10]$

Even if they could offer exquisite selectivities and affinities, the sensitivity of such constructs remains modest due the spectrophotometric properties of the reporter. In order to improve the detection limit, various groups have reported DAs based chemosensors relying on fluorescent indicators for various anions and applications. [4, 6-8, 11] The last development in that direction is an intramolecular IDA. [7]

Here, we report our efforts to develop a simple and easy to assemble fluorimetric chemosensor based on cerium (IV) combined with methyl calcein blue forming $[\mathrm{Ce}(\mathrm{MCB})]^{2+}[2]$ able to detect selectively phosphate anions with an FID in aqueous medium. The simple and cheap chemosensor is easy to assemble and composed of commercial chemicals, has high sensitivity due to fluorescence properties and excellent selectivity for phosphate ions over other anions, Finally, it shows high affinity for phosphate. In addition, we show, as an example, that our complex can be used to detect phosphate in Coca Cola $($ ).

\section{Materials and methods}

Dissolving methyl calceine blue (MCB, $250 \mu \mathrm{M})$ and cerium ammonium nitrate (CAN, $250 \mu \mathrm{M}$ ) in 2-[4-(2-hydroxyethyl)-1-piperazinyl]ethanesulfonic acid (HEPES, $10 \mathrm{mM}$, $\mathrm{pH}=7.4)$ formed $[\mathrm{Ce}(\mathrm{MCB})]^{2+}(250 \mu \mathrm{M})$, which is water-soluble and colorless at working micromolar concentrations (Figure 1). [12]

We hypothesized that $[\mathrm{Ce}(\mathrm{MCB})]^{2+}$ complex could be used to assemble a receptor for phosphate derivatives, exploiting metal-ligand interactions for selective target recognition. To the best of our knowledge, this mononuclear complex has never before been described in the literature as a selective FID-chemosensor for phosphate derivatives. We chose the reported methyl calcein blue (MCB), a fluorescent dye, as the fluorigenic indicator for the sensor, due to its described coordination to metal complexes, especially to lanthanides. [12]

$\mathrm{MCB}$ which is fluorescent at $\mathrm{pH}=7.4$ is quenched when coordinated to a metal. Therefore, the displacement of the receptor-bound methyl calcein blue by phosphate anions is communicated visually under the UV lamp (254 nm) as well as being readily measured spectrophotometrically via fluorescence (see figure 2). The competition assay developed is illustrated schematically in figure 1. The sensing ensemble was prepared by simply mixing CAN and MCB in a 1:1 molar ratio in an aqueous solution of $10 \mathrm{mM}$ HEPES buffer $\mathrm{pH}=7.4$, resulting in a non-fluorescent complex.

In the next step, we titrated CAN with MCB (visible as fluorescent blue) at $\lambda_{e x}=350$ 
A
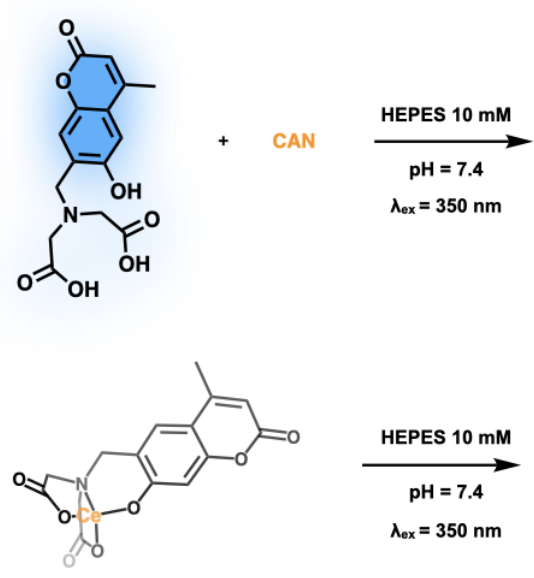

B

$\underset{\mathrm{pH}=7.4}{\stackrel{\text { HEPES } 10 \mathrm{mM}}{\longrightarrow}}$
$\lambda_{\mathrm{ex}}=350 \mathrm{~nm}$
B
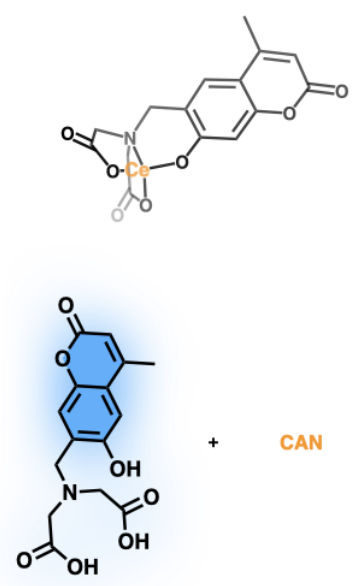

A

FIGURE 1: The proposed multistep reaction mechanism presenting the indicator displacement assay based on a fluorescent cerium complex for the dectection of phosphate in 10 $\mathrm{mM}$ HEPES $\mathrm{pH}=7.4$.

$\mathrm{nm}$ and $\lambda_{e m}=450 \mathrm{~nm}$. Out of the titration we conclude that the inflection point at 1 equivalent of CAN to MCB confirms the formation of a defined 1:1 non-fluorescent complex. The calculated affinity constant (see supporting information) is $3.98 \times 10^{4} \mathrm{M}^{-1}$, which is in good agreement with other similar described systems. [2, 12] The addition of phosphate anions to the aqueous solution of this violet ensemble resulted in the appearance of fluorescence (See Figure Figure 2).

The present sensor exhibits excellent selectivity towards phosphate ions over other anions, including pyrophosphate and sulfate ions (See Figure 3 ). To our knowledge, $[\mathrm{Ce}(\mathrm{MCB})]^{2+}$ is the first fluorometric sensor based on cerium to date that can detect phosphate ions with high selectivity in an aqueous medium. Sensors that can detect analytes by the fluorescent, is of particular interest due to their high sensitivity. The use of the present ensemble for such a purpose is demonstrated inFigure 3: the quenched fluorescence is recovered only when phosphate ion was added to the aqueous solution of the ensemble, whether all other anions tested failed to cause this remarkable fluorescent change even pyrophosphate (see Figure 3).

In order to determine the association constant of phosphate and the stoichiometry for $[\mathrm{Ce}(\mathrm{MCB})]^{2+}$, we carried out a titration (see supporting informations). At a 1:1 mixture $[\mathrm{Ce}(\mathrm{MCB})]^{2+}$ versus $\mathrm{PO}_{4}{ }^{3-}$ a maximum of fluorescence is reached and the calculated association constant of phosphate to cerium (IV) is $\mathrm{K}_{a s s}=3.98 \times 10^{7} \mathrm{M}^{-1}$, which 
a)

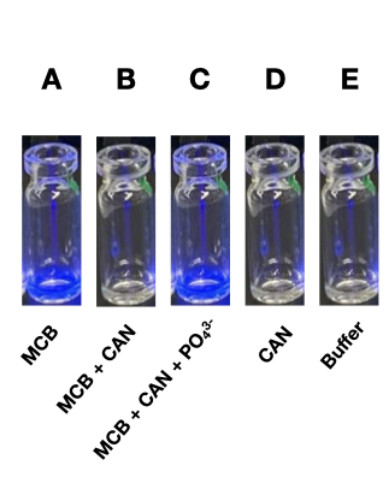

b)

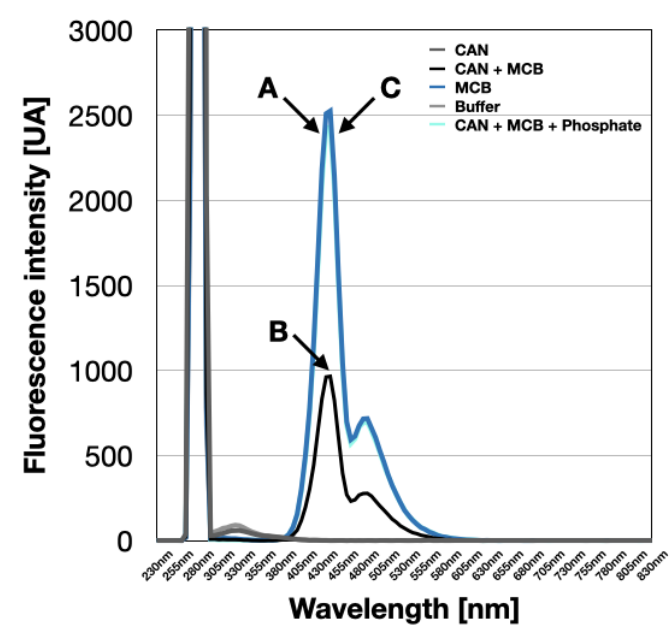

FIGURE 2: a) addition of methylcalcein blue (A, MCB, $250 \mu \mathrm{M})$ that is fluorescent under the UV lamp to cerium ammonium nitrate (CAN, $250 \mu \mathrm{M})$ allows quenching of the fluorescence, Exication scan optimum: $\lambda_{e x}=350 \mathrm{~nm}$ and emission scan optimum: $\lambda_{e m}=450$ $\mathrm{nm}$ b) Addition of phosphate $(250 \mu \mathrm{M})$ to the complex $[\mathrm{Ce}(\mathrm{MCB})]^{2+}$ allows an indicator displacement assay allowing liberation of MCB presented in the fluorescence spectras of the following vials at $\lambda_{e x}=280 \mathrm{~nm}$ and $\lambda_{e m}=450 \mathrm{~nm}$.

is at least 1000 times higher than similar described systems for detection of phosphatederivatives [5] and similar to a colorimetric dinuclear cerium sensor that we already reported in the literature. $[2,10]$ To our knowledge, this is to date the best simple system for selective naked-eye based phosphate recognition with a mononuclear cerium complex.

Finally, we investigated the possibility to detect phosphate in Coca Cola $\subset$. The literature gives a concentration of phosphoric acid in this drink of: $1.93 \mu \mathrm{M}$. [13] In order to do so, we diluted a sample of Coca Cola $\odot(50 \mu \mathrm{L}$ in $450 \mu$ HEPES $10 \mathrm{mM} \mathrm{pH}=7.4)$ and measured the appearance of fluorescence. Using a calibration curve we obtained a concentration of phosphate of: .It is similar to the described concentration in the literature.

\section{Conclusion}

In conclusion, we have described a simple assay for the detection of phosphate ions in an aqueous medium. The assay is based on commercially available chemicals (cerium salts and a fluorescent indicator), and it allows quantifying phosphate in the low micromolar 
a)

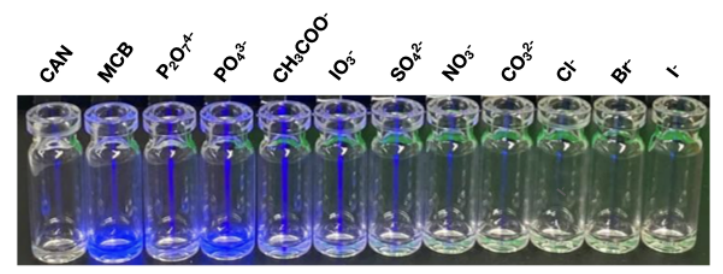

b)

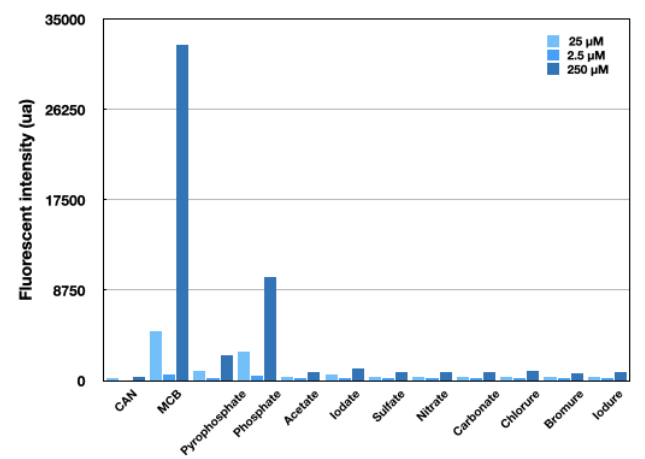

c)

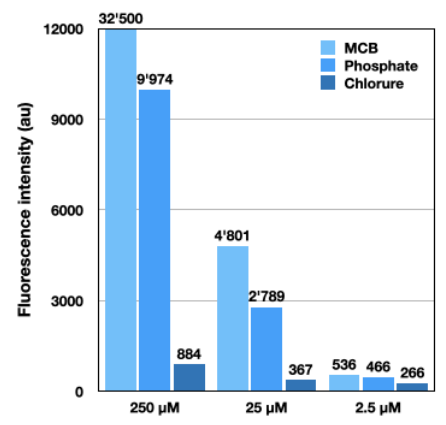

Figure 3: a) Screening of $[\mathrm{Ce}(\mathrm{MCB})]^{2+}(250 \mu \mathrm{M}$ in HEPES $10 \mathrm{mM}$ at $\mathrm{pH}=7.4$, aqueous solution) in the presence of various anions $(1.25 \mathrm{mM}, 5 \mathrm{x}$ excess) and exposed to a UV lamp at $254 \mathrm{~nm} \mathrm{~b}$ ) Fluorescence measurement of the mixture of $[\mathrm{Ce}(\mathrm{MCB})(\text { anions })]^{2+}(250 \mu \mathrm{M})$ : from left to right exposed to various anions; CAN, $\mathrm{MCB}, \mathrm{Na}_{2} \mathrm{P}_{2} \mathrm{O}_{7}, \mathrm{Na}_{3} \mathrm{PO}_{4}, \mathrm{CH}_{3} \mathrm{COONa}, \mathrm{NaIO}_{3}, \mathrm{NaSO}_{4}, \mathrm{NaNO}_{3}, \mathrm{Na}_{2} \mathrm{CO}_{3}, \mathrm{NaCl}, \mathrm{NaBr}$, $\mathrm{NaI}\left(\lambda_{e x}=350 \mathrm{~nm}\right.$ and emission scan optimum: $\left.\lambda_{e m}=450 \mathrm{~nm}\right) \mathrm{c}$ ) Detection limit of $[\mathrm{Ce}(\mathrm{MCB})]^{2+}$

concentration range by fluorescence measurements. The interference of common anions is remarkably low. With a detection limit of around $2.5 \mathrm{mM}$, our system is more sensitive than some of the best spectrophotometric methods described so far. However, it is very easy to perform and it requires no prior synthesis. The high affinity constant of cerium to phosphate in also the best described in the literature do date for a mononuclear complex. First evidence for the utility of our phosphate assay was provided by the successful analysis of phosphate in Coca Cola $@$ products.

\section{Acknowledgements}

We thank Prof. Thomas R. Ward for a loan of a spectrophotometer as well as Prof. Reinhard Neier for good advices in research. We thank the analytical service (NPAC) of the University of Neuchâtel.

Fig. X: Test band for the recognition of phosphate. Pieces of cellulosic paper where filled with $100 \mu \mathrm{L}$ of a solution of $[\mathrm{Ce}(\mathrm{MCB})]^{2+}$ at a concentration of $250 \mu \mathrm{M}$ and dryed 


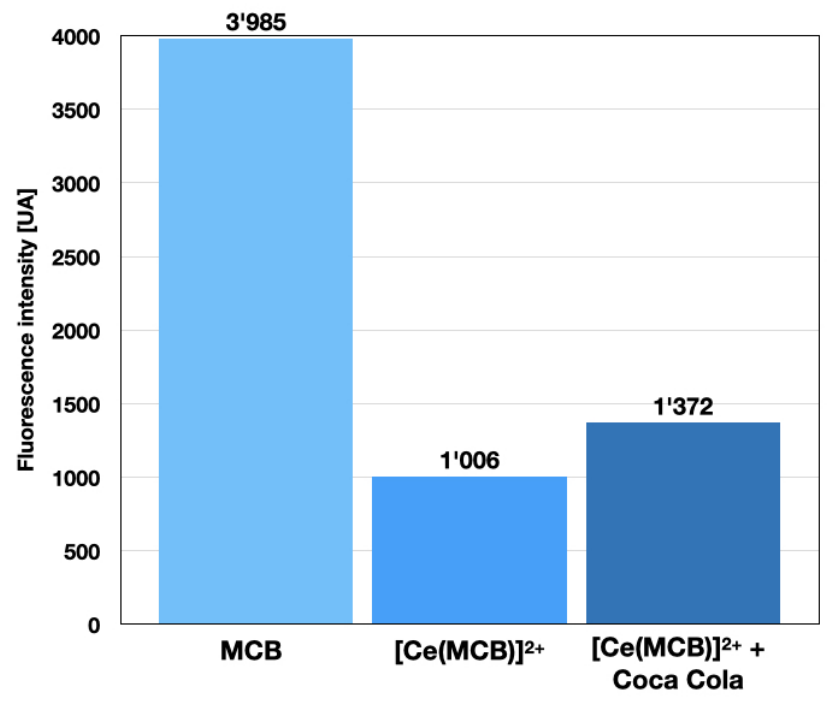

Figure 4: Detection of phosphate in Coca Cola $(C)$ with $[\mathrm{Ce}(\mathrm{MCB})]^{2+}\left(\lambda_{e x}=350 \mathrm{~nm}\right.$ excitation and $\lambda_{e m}=450 \mathrm{~nm}$ emission, gain 50)

overnight at $50^{\circ} \mathrm{C}$ in an oven. Dryed papers where exposed to $10 \mu \mathrm{L}$ of a $1.25 \mu \mathrm{M}$ solution of sodium phosphate.

\section{References}

[1] X. Gao, J. Xu, B. Ye, W. Wu, and H. Zheng, Analytical Methods, 2019, 11, 26772682.

[2] T. Rossel and M. Creus, Chemical Communications, 2019, 55, 14894-14897.

[3] A. E. Hargrove, S. Nieto, T. Zhang, J. L. Sessler, and E. V. Anslyn, Chemical reviews, 2011, 111, 6603-6782.

[4] J. Wu, B. Kwon, W. Liu, E. V. Anslyn, P. Wang, and J. S. Kim, Chemical reviews, 2015, 115, 7893-7943.

[5] M. S. Han and D. H. Kim, Angewandte Chemie International Edition, 2002, 41, 3809-3811.

[6] B. T. Nguyen and E. V. Anslyn, Coordination chemistry reviews, 2006, 250, 3118 3127. 
[7] A. C. Sedgwick, J. T. Brewster, T. Wu, X. Feng, S. D. Bull, X. Qian, J. L. Sessler, T. D. James, E. V. Anslyn, and X. Sun, Chemical Society Reviews, 2020.

[8] L. You, D. Zha, and E. V. Anslyn, Chemical reviews, 2015, 115, 7840-7892.

[9] N. Chatphueak and C. Suksai, Polyhedron, 2019, 170, 742-748.

[10] T. Rossel and M. Creus, CHIMIA International Journal for Chemistry, 2019, 73, 599-603.

[11] S. Rochat and K. Severin, Chemical Communications, 2011, 47, 4391-4393.

[12] H. G. Brittain, Analytical Chemistry, 1987, 59, 1122-1125.

[13] Découvrez les ingrédients des boissons Coca-Cola, 112020. 